\title{
Responsabilidad social y prevención de riesgos potenciales. Estudio de las grandes empresas españolas
}

\author{
Social responsibility and issues managent. Study of larg spanish \\ companies
}

\author{
Dra. Isabel Ruiz-Mora \\ Universidad de Málaga (España) \\ isabelruiz@uma.es \\ Dra. Silvia Olmedo-Salar \\ Universidad de Málaga (España) \\ silviaolmedo@uma.es
}

Recibido: 10 de septiembre 2013 Aceptado y Publicado: 24 de noviembre de 2013

\begin{abstract}
Resumen
El presente trabajo centra su atención en el papel que desempeña la responsabilidad social en la gestión proactiva de riesgos, desde la óptica de la comunicación y la relación con los públicos. Realizamos un análisis de las políticas de responsabilidad social y gestión de crisis de las grandes empresas españolas. Emplearemos una metodología basada en el análisis del contenido de la información relativa a las políticas de responsabilidad social y de gestión y prevención de riesgos que ofrecen las empresas seleccionadas en sus webs. Los resultados nos acercan a organizaciones que han avanzado en la implantación del enfoque de gestión responsable, pero que aún deben seguir trabajando en materia de gestión de riesgos y herramientas de participación de los grupos de interés.
\end{abstract}

\section{Abstract}

This paper is focused on the role of social responsibility in issues management from the perspective of communication and public relations. We analyze the social responsibility policies and crisis management of large spanish companies. The methodology is based on the content analysis of the information regarding to social responsibility policies and risk 
prevention offered by selected companies on their websites. The results bring us to organizations that have advanced in the implementation of a responsible management approach, but they still need to keep working on risk management and participation tools for stakeholders.

Palabras clave: Responsabilidad social; Crisis; Riesgos potenciales; Empresas españolas; Relaciones Públicas; Comunicación.

Keywords: Social responsibility; Crisis; Issues management; Spanish Companies; Public Relations; Communication.

\section{Introducción}

La situación actual que vivimos globalmente está provocando una acción y reacción en las empresas en materia de responsabilidad social y gestión de crisis y conflictos potenciales. La Responsabilidad Social de las Empresas (RSE) ${ }^{i}$ se ha convertido en una de las exigencia que los grupos de interés comienzan a interiorizar en sus demandas, no sólo los accionistas se ha convertido en reivindicadores de empresas que actúen conforme a unos principios y códigos de conducta, los consumidores demandan servicios producidos bajo uso de estándares de calidad y seguridad, los empleados valoran positivamente las medidas conciliadoras y la conducta ética de la empresa para la que trabajan, entre otros aspectos.

Con esta investigación pretendemos conocer la relación que puede existir entre un enfoque de gestión responsable y la prevención de riesgos en las organizaciones, conocer si el correcto establecimiento de políticas responsables puede servir como una herramienta proactiva para la detención de riesgos. Derivado del establecimiento de una correcta política de responsabilidad social, la gestión crisis y conflictos potenciales se verá reforzada por una actitud responsable y proactiva que repercutirá favorablemente, entre otros aspectos, en la actitud de nuestros públicos durante el trascurso de una crisis o en la capacidad de la organización para prevenir situaciones de riesgo con anterioridad, derivado de una correcta identificación de issues o asuntos relacionados con su entorno y grupos de interés.

El objeto de estudio de la presente investigación, se enmarca en el estudio de la Responsabilidad Social como una actitud proactiva para la prevención de riesgos potenciales en las organizaciones y nuestro objetivo general es conocer la relación existente entre la Responsabilidad Social y la prevención de crisis en las principales empresas españolas. Como objetivos específicos destacamos:

- Determinar cuáles son las iniciativas de Responsabilidad Social, nacionales e internacionales, que tienen una incidencia más directa en la gestión empresarial.

- Evaluar las estrategias de comunicación de las webs de las principales empresas españolas en materia de comunicación y prevención de crisis.

- Conocer la importancia que las grandes empresas otorgan a la relación con sus 
stakeholders, como elemento clave para la gestión de crisis en las estrategias empresariales.

\subsection{Gestión proactiva de riesgos y crisis}

La importancia que tienen las Relaciones Públicas en la organización no se limita únicamente a gestionar la relación con los accionistas o clientes, va mucho más allá, abarcando a toda la organización y centrando su interés en la relación con los públicos, de hecho si tomamos como referencia una de las definiciones más conocidas sobre Relaciones Públicas vemos cuál es el protagonismo de las mismas; la Asociación Internacional de Relaciones Públicas (IPRA) definen las Relaciones Públicas como:

La actividad de dirección de carácter permanente y organizado por la cual una empresa o un organismo público o privado, busca obtener o mantener la compresión, la simpatía y el concurso de aquellos con los que tiene o puede tener que verit.

Aunque comúnmente las funciones de las relaciones públicas se circunscriben en dos ámbitos, interno y externo, las funcionas van mucho más allá y profundizan en áreas de trabajo de los departamentos de comunicación, éstas están relacionadas con la imagen corporativa, comunicación interna, eventos y protocolo, relaciones institucionales, relaciones con los medios de comunicación, gestión de crisis y responsabilidad social, entre otras. Del conjunto de acciones comunicativas que se pueden realizar por parte de las organizaciones cabe destacar que la gestión de conflictos potenciales y las situaciones de crisis deben estar previamente planificadas, aunque aún existan organizaciones que dejen estas acciones en manos de la improvisación.

Desde el ámbito de la comunicación, entendemos por crisis a "una situación que amenaza los objetivos de la organización, altera la relación existente entre ésta y sus públicos, y precisa de una intervención extraordinaria de los responsables de la empresa para minimizar o evitar posibles consecuencias negativas" (González Herrero, 1998, p.30).

Toda crisis requiere de la intervención directa de la dirección, por lo que la identificación previa de los posibles riesgos es clave a la hora de reaccionar ante ellos. La correcta identificación y planificación suele aminorar considerablemente los efectos. Según ese mismo autor, podemos clasificar las crisis en función de las posibilidades de intervención que tiene la organización en ellas, distinguiendo aquellas que resultan evitables de las no evitables o accidentales (1998, p.36):

- Crisis evitables: acontecimientos que pueden evitarse mediante una intervención oportuna y eficaz. El fin principal sería evitarlas. La organización debe preverlas y adoptar medias proactivas que reduzcan sus posibles consecuencias. 
- Crisis no-evitables o accidentales: situación con origen normalmente externo a la organización, en agentes de la naturaleza, pero también en errores o acciones humanas no controlables por la empresa, como son los accidentes.

Como señala Capdevilla las crisis sirven para aprender, ya que "son oportunidades de aprendizaje y de rectificación. A través de la comunicación de los programas de crisis se enseña a prevenir y reparar el daño, a la vez que a recuperar la buena imagen de nuestra organización (2009, p.4).

El issues management, entendido como la gestión de conflictos potenciales, se convierte en un principio esencial que caracteriza la actitud proactiva de las organizaciones socialmente responsables, ligada íntimamente con la gestión de la comunicación de crisis. Según Chase ${ }^{\mathrm{iii}}$, el issues management se puede entender como:

La capacidad de comprender, movilizar, coordinar y dirigir todas las funciones estratégicas y de planificación de políticas, y todas la cualidades en asuntos públicos/relaciones públicas, para lograr un objetivo: una participación significativa en la creación de la política pública que afecta al destino personal e institucional.

Las organizaciones responsables que basan su relación con los grupos de interés en el diálogo y la transparencia, deben cumplir las expectativas de sus grupos de interés, asumir sus compromisos y evitar que sus actuaciones desemboquen en acontecimientos críticos que afecten su relación con la sociedad. Para ello, el departamento encargado de la política de RS deberá contar con un plan de prevención, o lo que Marín (2008) denomina "Plan de Continuidad de Negocio y de Prevención de Riesgos", un plan que concrete "las necesidades y acciones para garantizar la continuidad del negocio, mediante el análisis de impactos potenciales que puedan impactar a la entidad y adoptar las medidas necesarias para prevenir, afrontar y recuperarse de la activación de cualquier riesgo" (2008, p.209).

Basado en el enfoque de la RSE, las organizaciones contarán con un área de prevención formada por profesionales de la comunicación y de los principales departamentos susceptibles de sufrir una crisis. El equipo desarrollará un "mapa de riegos", donde se identifican los posibles riesgos que pudiera enfrentar la organización, y diseñarán un plan de planificación, prevención y reacción.

El Plan de Continuidad de Negocio y de Prevención de Riesgos integrará varios planes en torno a dos de las áreas de gestión de riesgos: la proactividad y la reactividad de la organización (Marín, 2008). La gestión proactiva contemplará las acciones anticipadas de gestión y prevención de riesgos, transmitiendo la filosofía de trabajo preventivo a todo el organigrama organizativo. La gestión reactiva abarcará los planes que diseñe e implemente la organización para la actuación antes los riesgos que se acontezcan y afecte a la organización, a sus públicos y al entorno. 


\subsection{Responsabilidad Social y gestión proactiva de riesgos}

Ofrecemos la definición de Responsabilidad Social promovida desde el Foro de Expertos del Ministerio de Trabajo y Asuntos Sociales del gobierno español (2008),

La responsabilidad social de la empresa es, además del cumplimiento estricto de las obligaciones legales vigentes, la integración voluntaria en su gobierno y gestión, en su estrategia, políticas y procedimientos, de las preocupaciones sociales, laborales, medioambientales y de respeto a los derechos humanos que surgen de la relación y el diálogo transparentes con sus grupos de interés, responsabilizándose así de las consecuencias y los impactos que se derivan de sus acciones.

En esta definición vemos como la voluntariedad por la RSE se integra en todos ámbitos de la empresa y se hace hincapié en la relación y el diálogo que toda empresa mantiene con sus grupos de interés. Un aspecto a destacar es la inclusión de la asunción de las responsabilidades que las empresas deben hacer de sus acciones. En este punto, la gestión de crisis se integra en la estructura de la responsabilidad social, las empresas que asumen las consecuencias de sus acciones y sus responsabilidades, están incidiendo positivamente en la prevención de futuras crisis o en la gestión de las que se estén produciendo.

Fruto del establecimiento de una política de gestión basada en un enfoque responsable, la identificación de los asuntos o issues ${ }^{\mathrm{iv}}$ se puede transformar en un proceso más ágil para la organización y viceversa. Las empresas que asumen la Responsabilidad Social como enfoque de gestión, se comprometen con la rendición de cuentas en materia de RSE y siguiendo la Triple cuenta de resultados, dicha rendición se materializa en una memoria anual. Uno de los estándares más conocidos son las directrices impulsadas por el Global Reporting Initiative (GRI) para la elaboración de memorias anuales de RS o sostenibilidad y su modelo permite que las organizaciones identifiquen sus propios indicadores más relevantes en el ámbito económico, medioambiental y social, basado en un enfoque multidisciplinar y multistakeholder. Por tanto, esta identificación de los indicadores, permitirá a la organización definir algunos de los asuntos que pueden llegar a afectar a la organización.

Como menciona González Herrero, en la gestión de conflictos potenciales hay que "identificar, mediante la vigilancia y el seguimiento de los acontecimientos del entorno, aquellos asuntos que pudieran afectar a la empresa en el futuro" $(1998$, p.124) y los indicadores de desempeño en Responsabilidad Social del GRI se centran en una dimensión económica, medioambiental (materiales; energía; agua; biodiversidad; emisiones, vertidos y residuos; productos y servicios; cumplimiento normativo; transporte, general) y social (prácticas laborales y ética del trabajo, Derechos Humanos, sociedad y responsabilidad 
sobre los productos) y éstas están relacionadas íntimamente con el entorno de la organización.

El establecimientos de una correcta política en materia de responsabilidad social y su correcto reporting, beneficiarán la labor de la identificación de los asuntos por parte de la organización, ya que antes de planificar las acciones de RS habrá que investigar lo que se está haciendo en la materia y lo que ocurre en nuestro entorno; e igualmente se facilitará información sobre la actividad de la organización en dichos ámbitos. Por tanto la organización estará actuando proactivamente ante cualquier riesgo evitable que pueda devenir de dichas dimensiones, ya que los está identificando y por tanto, actuará con anterioridad al posible conflicto.

De forma inversa, la identificación de los issues puede influir en el establecimiento de las políticas responsables de la organización. La definición de los posibles riesgos que pueden acontecer en una organización se puede realizar mediante el establecimiento de un plan de prevención, en el cuál a través de diferentes técnicas de investigación se pueden identificar los riesgos del entorno de la organización. La interpretación de la información suministrada por estas técnicas permitirá a la organización identificar los asuntos que pueden llegar a afectar a la organización, su capacidad de impacto y su priorización, fruto de esta identificación obtendremos un mapa de issues y por contrapartida, un mapa de los posibles ámbitos sensibles de unas políticas responsables.

Nos referimos a áreas de trabajo en las que la organización puede no estar trabajando desde el punto de vista de la RS y ser éstas, necesarias de dichas políticas, como pueden ser colectivos inmigrantes, violencia de género, comunidades afectadas por actividades de la organización, diálogo con la competencia,... Son asuntos desde los que pueden provenir posibles conflictos y crisis, pero que con una correcta gestión y establecimiento de acciones en materia de RS pueden dejar de ser una amenaza y convertirse en una oportunidad para la organización.

González Herrero, aborda la relación existente entre RS y la comunicación de crisis ${ }^{\vee}$ desde una triple perspectiva (2006, p.8):

- La gestión socialmente responsable de las empresas ayuda a solventar y evitar situaciones. A través del issues management la organización conoce su entorno con el fin de detectar posibles problemas en sus primeros síntomas.

- Las empresas con una imagen y reputación corporativa positiva suelen solventar las situaciones de crisis con un menor impacto negativo sobre su organización. Y siendo la responsabilidad social uno de los componentes, aunque no el único, que conforman la imagen y reputación de las empresas, parece lógico que exista cierta correlación entre el balance de responsabilidad social y la imagen y reputación de la organización. 
- La comunicación de crisis y la responsabilidad social corporativa pueden estudiarse desde la perspectiva del deber ético que tienen los directivos de las empresas para actuar de modo socialmente responsable ante las situaciones de crisis.

\subsection{Responsabilidad Social y Relaciones Públicas}

En algunas ocasiones, expertos, foros, iniciativas,... cuando hablan de la comunicación de la Responsabilidad Social se refieren al reporting, entendiendo por tal la elaboración de memorias anuales sobre RS, pero más que de comunicación, ésta es una herramienta informativa, las empresas informan sobre los que están haciendo en materia de RS. Es unidireccional, no hay ninguna interrelación con sus públicos.

Podemos decir que la comunicación en las organizaciones se convierte en un proceso básico que permite a los actores co-orientar sus conductas (Lucas, 1997, p.103), permite a los miembros de la organización, internos y externos, trabajar juntos hacia la consecución de sus fines. Se establecen acuerdos mutuos a través de relaciones comunicativas y la comunicación de convierte en el instrumento que se usa para establecer relaciones efectivas (Kreps, 1995, p.5) $)^{\mathrm{vi}}$. Por tanto, es de vital importancia, mantener una comunicación bidireccional con los grupos de interés de la organización, basado en el diálogo, la escucha y la participación.

Más allá del reporting, las organizaciones cuentan, gracias a los departamentos de comunicación, con las Relaciones Públicas y las nuevas tecnologías, con múltiples herramientas e instrumentos que facilitan la comunicación con sus públicos. La gestión de las relaciones con los públicos se postula como una de las principales funciones de las Relaciones Públicas (Harlow, 1975 vii; Seitel, 2002; Grunig y Hunt, 2003) y éstas, se convierten en un elemento necesario para la correcta gestión y desarrollo de la RSE; las Relaciones Públicas se transforman en un elemento cohesionador de la RSE al relacionar a la organización con sus stakeholders.

Abordar la gestión de la RSE desde las Relaciones Públicas parece algo natural, debido a la importancia de las relaciones estratégicas que las organizaciones deben establecer con sus públicos al diseñar su política de RSE (Oliveira y Nader, 2006, p.104) y la gestión de las relaciones con los stakeholders, está tanto en el corazón de la RSE como en las funciones de las Relaciones Públicas (Wang y Chaudhri, 2009, p.247).

Aunque no entraremos en detalle a definir y comentar las funcionalidades y beneficios de todas las posibilidades, comentamos las estrategias, acciones y herramientas comunicativas que tienen una mayor influencia en la gestión de riesgos. Entre muchas posibilidades nos focalizaremos en tres aspectos clave:

- Comunicación de Crisis. La organización deberá establecer una estrategia de comunicación en crisis. En este apartado la organización contará con un Plan de 
Comunicación para situaciones de crisis, con un gabinete de crisis y un portavoz; si las situaciones de crisis se gestionan desde otro departamento diferente al de comunicación, éste debe mantener una correcta y fluida comunicación con el área responsable. No podemos olvidar la formación especializada y el diseño de instrumentos normalizados para la gestión de una crisis.

- Públicos. La organización de conocer a sus públicos, internos y externos, realizar una correcta segmentación de sus stakeholders y establecer una estrategia adecuada a cada uno de ellos, en función de los objetivos de la organización. Se debe mantener una comunicación bidireccional que nos permita conocer sus demandas y necesidades, para ello las herramientas 2.0 nos facilitan el diálogo y la comunicación. Aunque como mencionan en su trabajo

- Estrategia y Plan de Comunicación General. Independientemente del plan de comunicación de crisis, la organización debe contar con un Plan de Comunicación y una estrategia comunicativa general, que armonice todas las acciones comunicativas y relaciones con los grupos de interés que se producen en el día a día de la organización.

Prestando especial atención a la relación de los públicos y la gestión de crisis con las herramientas 2.0, tomamos el trabajo de Domínguez, Álvarez y Martí sobre la Dirección de Comunicación en Internet y donde afirman que:

A día de hoy la empresa no ha resuelto con claridad si le interesa esta comunicación (2.0) más abierta y transparente, presentando unos portales corporativos activos pero aún lejos del primer plano de la dirección de comunicación, donde sigue primando la gestión de la imagen, la publicidad y la relación con los medios de comunicación (2012, p.51).

\section{Metodología}

Para analizar el carácter preventivo que las organizaciones asumen al apoderarse del enfoque de Responsabilidad Social como enfoque de gestión, procederemos a realizar un análisis de las políticas de responsabilidad social y gestión de crisis de las grandes empresas españolas.

Basados en el método hipotético-deductivo, emplearemos una metodología basada en un estudio del contenido que las empresas seleccionadas ofrecen en su página web, en la sección que contiene la información relativa a las políticas de Responsabilidad Social y de gestión y prevención de riesgos, así como de los informes de RSE a los que podemos acceder a través del site empresarial. Estudiaremos, desde un punto de vista proactivo, la información que ofrecen las empresas en sus webs, considerando que las empresas que comunican públicamente las medidas seguidas en materia de RS, están asumiendo un 
enfoque responsable en su gestión y por tanto está incidiendo positivamente en la prevención de riesgos y en la imagen que los stakeholders tienen sobre ellas.

Para llevar a cabo nuestra investigación se ha seleccionado una muestra compuesta por las 10 primeras empresas cotizadas en el IBEX 35 (Iberia Index), el índice de referencia bursátil de la Bolsa Española, elaborado por Bolsas y Mercados Españoles.

La selección de la muestra se ha basado en el hecho de que estas diez empresas, son las empresas españolas de un mayor tamaño, mayores ingresos y mayor volumen de negocio dentro de la Comisión Nacional del Mercado de Valores (CNMV). Son empresas con una gran número de grupos de interés, además tienen que satisfacer las demandas fiscales y sociales que le exige la sociedad y como una de la respuestas a estas demandas, cuentan con las políticas en materia de RS; por tanto, invertirán una mayor cantidad de recursos financieros en Responsabilidad Social, llevarán un mayor tiempo desarrollando un enfoque de gestión basado en la Responsabilidad Social y nos ofrecerán una información más fiable sobre la relación y la importancia que ellas le otorgan a este enfoque para la prevención de riesgos.

Tabla 1. Corpus de estudio

\begin{tabular}{|l|l|l|}
\hline Empresa & Capitalización $\mathbf{-} \boldsymbol{\text { P }}$ & Ponderación \\
\hline Santander & $96.729 .851 .216,9250$ & $23,33 \%$ \\
\hline Telefónica & $84.456 .754 .954,9250$ & $20,37 \%$ \\
\hline BBVA & $48.929 .736 .874,6550$ & $11,80 \%$ \\
\hline Iberdrola & $35.059 .260 .804,3000$ & $8,45 \%$ \\
\hline Repsol YPF & $22.793 .520 .854,2100$ & $5,50 \%$ \\
\hline Inditex & $16.289 .493 .343,2000$ & $3,93 \%$ \\
\hline Arcel. Mittal & $9.323 .197 .530,0750$ & $2,25 \%$ \\
\hline Acs Const. & $9.052 .271 .033,0000$ & $2,18 \%$ \\
\hline Abertis Infr & $8.631 .961 .303,9800$ & $2,08 \%$ \\
\hline Banco Popular & $7.646 .958 .093,8400$ & $1,84 \%$ \\
\hline
\end{tabular}

Fuente: http://www.ibex35.com ${ }^{\text {vi }}$

El acceso estará materializado en cada una de las webs corporativas de las empresas que comprenden nuestra selección ${ }^{\text {ix }}$.

\subsection{Técnica de investigación}

Como se ha mencionado, la técnica de investigación seleccionada ha sido el análisis de contenido. Piñuel describe el análisis de contenido como un: 
Conjunto de procedimientos interpretativos de productos comunicativos (mensajes, textos o discursos) que proceden de procesos singulares de comunicación previamente registrados, y que, basados en técnicas de medida, a veces cuantitativas (estadísticas basadas en el recuento de unidades), a veces cualitativas (lógicas basadas en la combinación de categorías), tienen por objeto elaborar y procesar datos relevantes sobre las condiciones mismas en que se han producido aquellos textos, o sobre las condiciones que puedan darse para su empleo posterior (2002, p.2).

Otros autores, como Krippendorff, señalan que análisis de contenido

Debe ser predictivo de algo en principio observable, que facilite la toma de decisiones o que contribuya a conceptualizar la porción de la realidad que dio origen al texto analizado. Cualquier análisis de contenido debe realizarse en relación con el contexto de los datos (1990, p.32).

Esta técnica nos facilitará el estudio de la existencia o no de los elementos que nos indiquen la actitud preventiva en las organizaciones responsables y nos permitirá acercarnos a nuestro objeto de estudio, para poder obtener unas conclusiones caracterizadas por la fiabilidad y la veracidad de los resultados derivados del análisis.

De acuerdo con la técnica de investigación seleccionada, se ha desarrollado una ficha de análisis en la que se establecen las variables e indicadores que pronostican si la organización mantiene un comportamiento proactivo. Estos índices se han agrupado en tres grupos, (i) gestión, (ii) comunicación e (iii) iniciativas de apoyo a la RS en la organización. Analizaremos la presencia e inclusión de estos indicadores en la web corporativa y en los informes de responsabilidad social. A continuación detallamos cada indicador.

\section{a) Gestión}

En este apartado se verificará la presencia de diferentes elementos que incidan en la prevención de riesgos de la gestión que se lleva a cabo en el seno de la organización. Gracias a este apartado conoceremos qué hace la entidad para incidir positivamente en la prevención. Analizamos la presencia de:

1. Códigos Éticos, de Conducta o Declaración de Valores Corporativos (información contra sobornos, competencia desleal, fraude, anticorrupción). Tanto de para la propia empresa como para proveedores.

2. Políticas de RS que se promueven desde la empresa. Identificamos las principales políticas en materia de RS

3. Indice Bursátil de Sostenibilidad en los que la empresa está presente

4. Departamento o Área de Gestión y Prevención de Riesgos (denominación; si es un área específica, ver en qué departamento se integra y posición en el organigrama de 
la organización). Vemos en qué estado se encuentra el área encargada de la gestión de riesgos.

5. Mapa de Riesgos. Identificación de los riesgos a los que la empresa puede tener que hacer frente.

6. Plan de Prevención de Riesgos, como un elemento claro de proactividad.

7. Plan de Gestión Crisis, información para saber cómo actuar en las situaciones de crisis que se produzcan.

8. Formación a la plantilla en materia de Prevención y Gestión de Riesgos.

b) $\underline{\text { Comunicación }}$

En este apartado, estudiamos la estrategia de comunicación que la empresa sigue como organización responsable, cómo comunica su estrategia. Aunque la comunicación es intrínseca a cualquier organización, y por tanto deben comunicar todas las acciones y decisiones que toman, estudiaremos específicamente:

1. Estrategia de Comunicación en Crisis. Plan de Comunicación para situaciones de Crisis. Relación del Departamento de Comunicación con el área o departamento de Prevención y Gestión de Riesgos.

2. Segmentación e identificación de los stakeholders. Este indicador nos permite conocer si la empresa tiene delimitados sus públicos, conoces sus demandas y les da respuesta.

3. Estrategia de comunicación segmentada para cada stakeholder. Estudiamos si ofrecen información especializada y adecuada a las necesidades de cada grupo de interés, si se fomenta el diálogo y la participación.

4. Herramientas de Comunicación 2.0. que faciliten el diálogo y la comunicación con los grupos de interés.

5. Estrategia y Plan de Comunicación General (independientemente del plan de comunicación de crisis). Este indicador nos permitirá conocer como se integra el plan de comunicación en crisis en la estrategia general de comunicación.

c) Iniciativas de apoyo a la Responsabilidad Social

Esta sección nos ayudará a conocer los esfuerzos que la organización realiza para promover la RS como modelo de gestión empresarial y por consecuencia, como modelo de prevención de crisis en las empresas.

1. Iniciativas Internacionales que apoya la organización (Adhesiones)

- El Pacto Global (The Global Compact), persigue conseguir un compromiso voluntario de las organizaciones en responsabilidad social, por medio de la aceptación e implantación de Diez Principios basados en derechos humanos, laborales, medioambientales y de lucha contra la corrupción.

- Líneas Directrices de la Organización para la Cooperación y el Desarrollo Económico (OCDE) para multinacionales, donde se enuncian unos principios y 
normas voluntarias para una conducta empresarial responsable compatible con las legislaciones aplicables en cada país.

- Declaración Tripartita de principios sobre las empresas multinacionales y la política social de la Organización Internacional del Trabajo. Tiene por objeto fomentar la contribución positiva que las empresas multinacionales pueden aportar al progreso económico y social, minimizando los posibles efectos negativos de sus operaciones.

- Pertenencia al Foro de Reputación Corporativa (fRC). Este foro está formado por empresas que dan una gran importancia a la gestión de intangibles (marca, reputación corporativa, ética empresarial, responsabilidad social corporativa, buen gobierno, capital humano, capital organizativo o capital tecnológico...) como generación de valor para las compañías y sus grupos de interés.

- Certificaciones (medioambientales, calidad, del proceso de investigación, desarrollo e innovación).

2. Proyectos y campañas que apoya la organización

3. Realización del informe de RS en base a los siguientes principios:

- GRI (Guía del Global Reporting Initiative) para la definición del contenido y verificación de la calidad de la Memoria de Responsabilidad Social.

- Norma AA1000/AA1000AS, para verificar la correcta relación de los contenidos de la memoria con los intereses de los stakeholders.

- Presentación del Informe de Sostenibilidad auditado de acuerdo con el nivel de aseguramiento limitado, establecido por la Norma ISAE 3000 con respecto a la aplicación de los criterios de la norma AA1000 AS y de la Guía de Global Reporting Initiative (GRI). En este caso, hablamos de una verificación independiente y externa de la memoria de RSE.

\section{Resultados}

La inclusión de declaraciones de principios, códigos de conducta y valores corporativos en las webs e informes sobre RS de las empresas analizadas transmite el valor que la transparencia y buen gobierno tiene no sólo para la organización, sino para los grupos que se ven afectados directamente por la gestión y trabajo de la organización empleados, accionistas e inversores, clientes y proveedores entre otros); esta información puede ser interpretada como una respuesta a las demandas informativas de los públicos, sinónimo de transparencia. Tratando la importancia de la transparencia en las organizaciones, nos gustaría destacar el hecho de que algunas de las organizaciones estudiadas (30\%), hablan claramente sobre los resultados económicos que les reporta las políticas en materia de RS, así como las investigaciones que hacen en esta materia (40\%). Como vemos esta información es claramente proactiva para la organización, informa sobre lo que hacen en RS, más allá del discurso y contribuyen en parte a mejorar la transparencia de la organización, incidiendo en parte en la confianza de sus públicos. 
Las políticas de responsabilidad social que se llevan a cabo en la organización son comunicadas en el $100 \%$ de los casos. Entre las políticas destacamos: formación de empleados; políticas de igualdad y conciliación; RR.HH.; DD.HH.; impacto ambiental; acción social (100\%); fundación/obra social (90\%); ética y gobierno corporativo (80\%); diálogo con stakeholders (60\%) e ISR (50\%).

Así mismo, la cotización de la organización en los índices bursátiles de sostenibilidad, nos ofrecen información sobre como las buenas prácticas sociales y medioambientales son reconocidas por agentes externos a la empresa, vemos que las empresas no se preocupan por ser rentables económicamente únicamente, hablamos de demostrar el desempeño que hace la organización en materia de RS y la presencia en estos índices lo demuestra. Esta decisión de la organización de cotizar en índices de sostenibilidad índice positivamente en las decisiones de muchos inversores en materia de inversión sostenible. Destacamos que el $90 \%$ de las empresas estudiadas están presentes en el índice bursátil Dow Jones Sustainability Index y el 60\% además, en FTSE4Good IBEX.

En el ámbito de la gestión de la Responsabilidad Social y la prevención de riesgos en la organización, este estudio nos ha permitido corroborar algunas inquietudes iniciales. Efectivamente las empresas identifican sus riesgos y los comunican, incluso algunas informan sobre los planes de prevención y gestión del riesgo que desarrollan, pero escasamente comunican cómo enfrentan esas situaciones desde el punto de vista de la comunicación. Este estudio no profundiza en la existencia 0 no de los planes de comunicación, sino en la comunicación que se realiza sobre los mismos, sólo el $20 \%$ refleja en sus web la estrategia de comunicación que sigue en materia de crisis. Esta carencia simplemente refleja una ausencia de proactividad, ya que el correcto diálogo con los stakeholders incide directamente con la mejora de las relaciones que mantenemos con ellos y cómo no, con nuestra reputación. Un ejemplo claro son los futuros proveedores o colaboradores de la organización, el conocimiento de la política comunicativa de la organización les ayudará en la toma de decisiones, gestión del negocio y estrategia comunicativa de cara a establecer contactos con la organización. Sin olvidar a los stakeholders del tercer sector y a los medios, ellos tienen una especial sensibilidad hacia la comunicación que se desprende de la organización.

En cuanto al departamento o área de gestión encargado de la prevención y gestión de riesgos, es remarcable su ausencia, apareciendo sólo en el $20 \%$ de los casos e integrado en otras áreas del organigrama de la organización. Por otro lado, el $80 \%$ ofrece información sobre los riesgos de la compañía (mapa de riesgos), así como plan de prevención de riesgos $(60 \%)$, sólo el $40 \%$ cuenta con un plan de crisis, pero en el $80 \%$ de los casos se ofrece formación a la plantilla en prevención y gestión de riesgos, aunque muy ligado al ámbito laboral y de seguridad. El $100 \%$ de las empresas cuentan con códigos de conducta para proveedores; este resultado deja ver cómo las empresas toman conciencia de su 
responsabilidad en la cadena de valor y comienzan a exigir responsabilidades a otros actores sociales implicados en su actividad.

En materia de formación, podemos decir que poco a poco las organizaciones introducen planes de formación entre sus empleados orientados a difundir el enfoque que sigue la organización en materia de Responsabilidad Social y la identificación y gestión de riesgos, no sólo entre los empleados sino entre un nutrido grupo de directivos y cargos corporativos, esta actitud incide positivamente en el futuro de la organización, ya que un empleado con conocimientos sobre gestión de riesgos puede identificar situaciones críticas para la organización evitando futuras crisis y sus efectos en la organización.

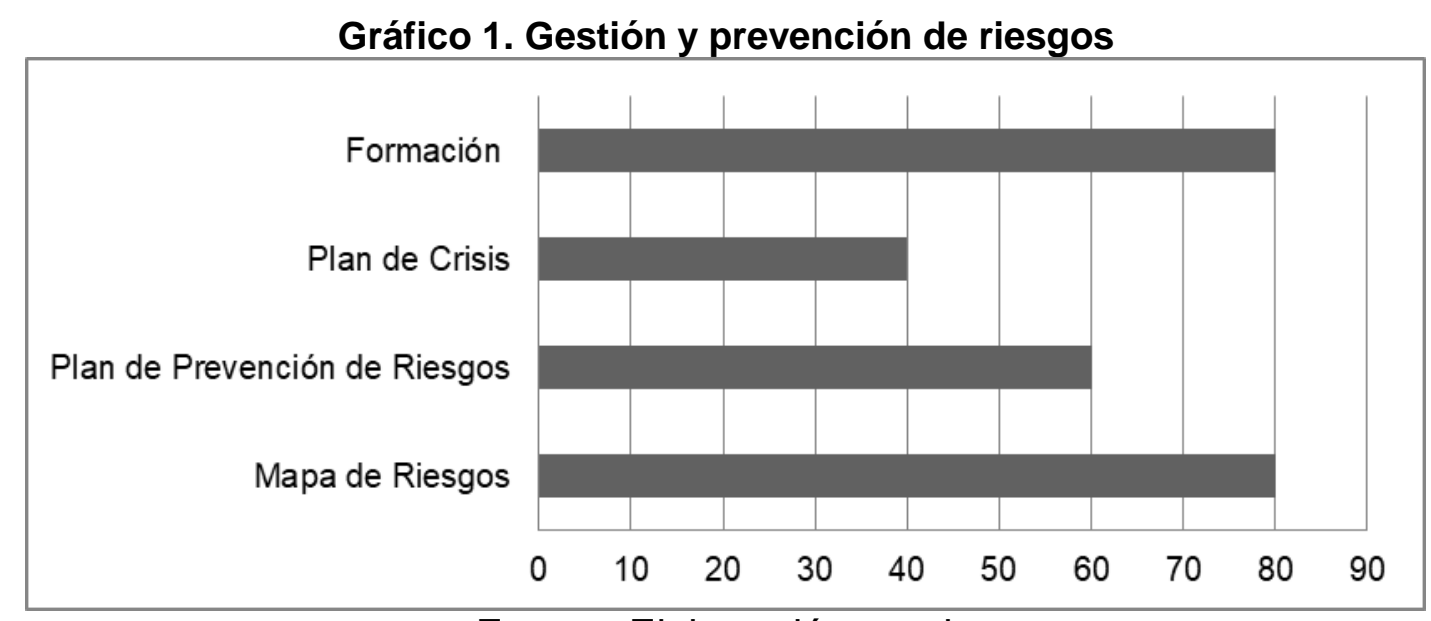

Fuente: Elaboración propia

Las empresas analizadas, en el $100 \%$ de los casos, identifican claramente a sus stakeholders y desarrollan políticas, iniciativas y estrategias de comunicación (el 90\%) adecuadas a cada uno de ellos. Esta predisposición por parte de la organización, nos transmite su interés por conocer a sus públicos, dar respuesta a sus demandas y mantener un diálogo abierto. A continuación ofrecemos un listado de los stakeholders identificados en el estudio: 
Tabla 2. Stakeholders

\begin{tabular}{|c|}
\hline Empleados \\
\hline Accionistas \\
\hline Proveedores \\
\hline Acreedores \\
\hline Colaboradores \\
\hline Propietarios \\
\hline Medios de Comunicación \\
\hline Comunidad Local \\
\hline Clientes \\
\hline Organismos Reguladores \\
\hline Inversores \\
\hline Medio Ambiente \\
\hline
\end{tabular}

Fuente: Elaboración propia

Comunicación bidireccional y herramientas 2.0, este indicador nos ha verificado la escasa presencia de estas herramientas entre las grandes empresas. La herramienta que tiene una mayor presencia es la personalización de noticias para cada stakeholder, que aunque no es puramente 2.0, esta herramienta nos ayuda a identificar la importancia del diálogo con los stakeholders, pero el resultado desanima. Estando presente en el $80 \%$ de los casos estudiados, el único stakeholder al que dirigen sus noticias es el sector prensa, por lo que la utilidad de esta herramienta es bastante limitada ya que sólo se utiliza para dirigirse a los medios de comunicación.

La sindicación de contenidos (RSS) es la herramienta con una mayor utilidad, ya que facilita la actualización de la relación con la organización no sólo a los medios, sino también a accionistas, inversores, clientes, ONG's,... muy ligada a esta noticia es el acceso a la documentación multimedia (imágenes, vídeos, entrevistas,...). El resto de herramientas son protagonistas por su escasa presencia (chats, foros, enlaces a redes sociales,...).

Fruto de esta escasa importancia otorgada a las herramientas 2.0 la relación con los stakeholders se ve claramente afectada, porque aunque la organización está dispuesta a mantener un diálogo abierto y participativo no ofrece todas la herramienta participativas que internet pone a nuestra disposición. Esta postura puede estar motivada -pero no justificadapor los procesos burocráticos que existen en las grandes organizaciones así como, la reticencia de éstas al cambio, a adaptarse a la nueva realidad de la sociedad en la que se integran. Esta adaptación puede suponer un cambio en la cultura corporativa asumiendo un nuevo modelo basado en la participación y colaboración y en un menor funcionamiento estructurado y estanco. 


\section{Gráfico 2. Herramientas 2.0}

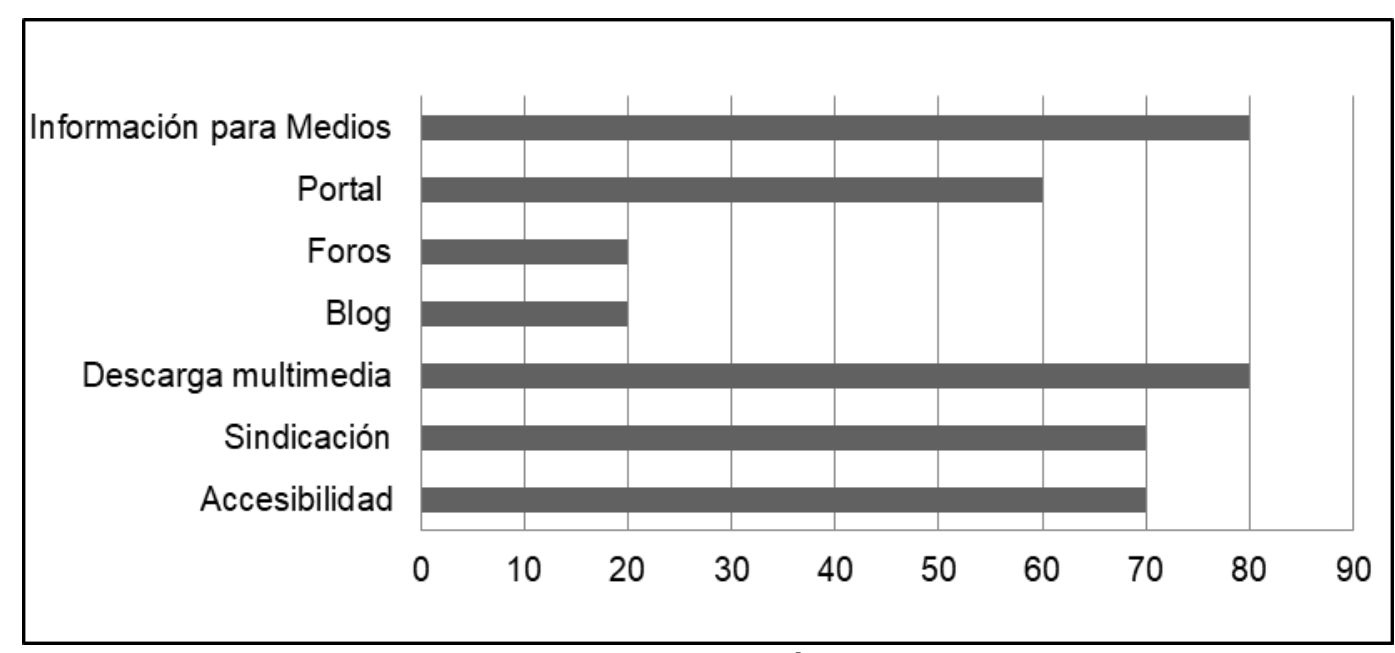

Fuente: Elaboración propia

La comunicación de las iniciativas de apoyo de la RSE índice positivamente en la imagen de la organización entre sus stakeholders, ven como las empresas asumen voluntariamente un compromiso internacional a favor de la responsabilidad social, los derechos humanos, sociales y medioambientales, trabajan en contra de la corrupción y se promueve la inversión socialmente responsable. En el $100 \%$ de los casos se comunica las adhesiones al Pacto Mundial de la Naciones Unidas, que siguen las Líneas Directrices de la OCDE y las de la Declaración Tripartita de la OIT, así como el respeto a los Derechos Humanos en su gestión.

La importancia que tiene para algunas empresas estar presente en el Foro de Reputación Corporativa, nos traslada la importancia que tiene para la organización la gestión de intangibles como generadores de valor también para sus stakeholders. Una empresa que se preocupa por su marca, la reputación entre sus públicos, su capital humano, transmite esta importancia e interés a su cadena de valor y por tanto a sus stakeholders. Destacamos la participación del $50 \%$ de las empresas en el Foro de Reputación Corporativa (fRC) de España; el apoyo a la campaña a favor de los Objetivos de Desarrollo del Milenio (70\%). Esta posición de la organización demuestra su grado de implicación con las preocupaciones de la sociedad, no sólo con su reputación, aunque igualmente podrían ser mayores al trabajar con organizaciones comprometidas con la sociedad.

La importancia que otorgan las empresas a los procesos de certificación nos trasmite que lo más importante es comunicar que se es responsable con el medio ambiente y la calidad; los resultados nos trasladan que la norma ISO 14001, referente al sistema de gestión medioambiental, se sigue en el $90 \%$ de los casos, la norma ISO 9001 que verifica el sistema de gestión de calidad, en el $60 \%$, y la norma UNE 166002, sobre certificación del proceso de investigación, desarrollo e innovación, en el 10\%. 
Aunque es positiva la actitud de las empresas al seguir procesos de certificación, no deben olvidar que éstos comunican y comunican lo que realmente es importante para ellas.

En lo referente a la elaboración de la memoria o informes anuales de RS, destacamos que el $90 \%$ de los casos la organizaciones se someten a verificaciones externa, que certifican que realizan la memoria siguiendo las indicaciones de GRI (100\%), verificando el contenido y calidad de la memoria, junto con las directrices de la Norma AA 1000 Accountability, para verificar la correcta relación de los contenidos de la memoria con los intereses de los stakeholders, y presentado con el nivel de aseguramiento de la Norma ISAE 3000, que verifica la aplicación de los criterios de la norma AA1000 AS y de la Guía de Global Reporting Initiative (GRI), en el $70 \%$ de los casos. Facilitan a los stakeholders la posibilidad de consultar los comentarios, consejos y certificaciones de los auditores externos y tomar éstos como una garantía de veracidad, transparencia y confianza hacia la organización.

\section{Discusión y conclusiones}

La evolución de la valoración de los públicos durante el trascurso de una crisis, dependerá en mayor o menor grado de la eficacia de la organización a la hora de gestionar una crisis, si ésta cumple con las expectativas de sus stakeholders, saldrá reforzada de la crisis, en el caso contrario, le afectará negativamente. En este punto retomamos las palabras de Arpan $(2005)^{x}$ que sugiere que "la información negativa acerca de una empresa -como la que se suele derivar de una situación de crisis- puede no ser perjudicial (...) en aquellas organizaciones que gozan de una fuerte reputación corporativa". Por lo tanto, la combinación de RS, issues management y comunicación actúa proactivamente en las organizaciones y les benefician en los momentos de crisis, si ese trío se ha gestionado adecuadamente.

La situación social basada en múltiples actores organizativos, grupos de interés divergentes, complejidad del proceso productivo y públicos cada vez más activos hace imprescindible gestionar proactivamente las organizaciones. Por ello, uno de los elementos de protección ante las situaciones de crisis pueden ser las acciones y políticas en materia de responsabilidad social que desarrollan las organizaciones, como enfoque de gestión, y las políticas comunicativas que se establezcan en esta materia.

Con el desarrollo de acciones de responsabilidad social podemos disponer de aspectos amortiguadores de las situaciones de crisis y disponer de una consideración social que nos ayude a responder adecuadamente a las situaciones conflictivas. Las situaciones de crisis se caracterizan por la imprevisibilidad y la exposición negativa de la organización ante la opinión pública, por lo que contar con un público predispuesto hacia la organización reducirá los efectos negativos que se pueda deducir de esta situación. De ahí la importancia de segmentar a nuestros stakeholders y diseñar estrategias comunicativas adecuadas a sus necesidades. 
Fruto de esta identificación de los stakeholders, surge la identificación de los riesgos a los que se enfrenta la organización, ya no sólo económicos o medioambientales, sino también sociales, reputacionales y de imagen, éstos relacionados estrechamente con el modelo de gestión que siga la organización, responsable o no, y su visión ante los riesgos, proactiva o reactiva. Como vemos la opción empresa responsable con una visión proactiva, es la que posibilita que la organización sea capaz de afrontar la situación actual, caracterizada por un trabajo previo basado en el diálogo con los públicos, la gestión responsable y el issues management.

El diálogo con los públicos, debe verse enriquecido por la nueva realidad de la red, la participación, la colaboración, el intercambio, la comunicación 2.0, basado eso sí en una sólida estrategia de comunicación asentada en el estudio de sus características y necesidades. Se debe contar con las memorias de sostenibilidad como uno de los cauces de relación con los grupos de interés, por la información que ellas proporcionan, pero nunca debe ser el único medio de comunicar la RSE de la organización, ya que entonces la comunicación se convertiría en unidireccional y no existirá un enfoque multiestakeholder.

Hablamos de gestión responsable, asumiendo un enfoque de gestión de la Responsabilidad Social en toda la organización, no sólo interesa a los accionistas, inversores, ONG's y medios, se debe trasladar al día a día de la organización, a todos los públicos.

Cuando adquirimos un enfoque de gestión responsable, la gestión de riesgos potenciales (issues management) se integra como elemento clave de prevención de situaciones críticas derivadas de la actuación de la organización y de la sociedad con la que se integra.

\section{Referencias}

Caldevilla Domínguez, D. (2009). Comunicar en situaciones de crisis. Vivat Academia, 105, 1-27. http://www.ucm.es/info/vivataca/anteriores/n105/DATOSS105.htm

Castillo, A. (2009). Relaciones Públicas. Teoría e Historia. Barcelona: UOC.

Dominguez Quintas, S., Álvarez Rodriguez, M.L. y Martí Pellón, D. (2012). Dirección de Comunicación en internet. Estudio y recomendaciones para los espacios de prensa en webs corporativas desde el análisis de portales en internet de grupos empresariales en Galicia. Revista Internacional de Relaciones Públicas, 3(2), 45-70. http://dx.doi.org/10.5783/RIRP-32012-03-45-70.

Foro de Expertos en RSE. (2007). Informe del Foro de Expertos en RSE. Consultado el 12 de de febrero, 2013. Disponible en http://www.mtas.es/es/empleo/economiasoc/RespoSocEmpresas/docs/INFORME FOROEXPERTOS RSE.pdf. 
Global Reporting Initiative. (2006). Guía para la elaboración de Memorias de Sostenibilidad. Consultado el 9 de febrero, 2013. Disponible en https://www.globalreporting.org/resourcelibrary/Spanish-G3-Complete.pdf

González, A. (1998). Marketing preventivo. La comunicación de crisis en la empresa. Barcelona: Bosch.

González Herrero, A. (2006). Comunicación de Crisis y Responsabilidad Social Corporativa. En Capriotti, P. y Garrido, F.J. (eds.), Guía de la Responsabilidad Social Empresarial (RSE), Fascículo 4 (pp. 8-11). Santiago (Chile): Universidad del Desarrollo/Diario Financiero.

Grunig, J. E. \& Hunt, T. (2003). Dirección de Relaciones Públicas. Barcelona: Gestión 2000.

Krippendorff, K. (1990). Metodología de análisis de contenido. Teoría y práctica. Barcelona: Paidós lbérica.

Losada, J. (2010). Comunicación en la gestión de crisis. Lecciones prácticas. Barcelona: UOC.

Seitel, F. (2002). Teoría y práctica de las Relaciones Públicas. Madrid: Pretencie Hall.

Lucas, A. (1997). La Comunicación en la empresa y en las organizaciones. Barcelona: Bosch.

Oliveira, M. J. Da Costa \& Nader, S. (2006). Relações Públicas na gestão da Responsabilidade Social: desafio e oportunidade. Organicom, 5, 2o Semestre, 97-107 http://www.revistaorganicom.org.br/sistema/index.php/organicom/article/view/73/206

Piñuel Raigada, J. L. (2002). Epistemología, metodología y técnicas del análisis de contenido. Estudios de Sociolingüística, 3 (1), 1-42.

http://www.ucm.es/info/mdcs/A.Contenido.pdf

Wang, J. \& Chaudhri, V. (2009). Corporate social responsibility engagement and communication by Chinese companies. Public Relations Review, 35, 247-250. 
i Para Referirnos a la Responsabilidad Social de las empresas utilizaremos la abreviatura RSE y cuando hablemos de Responsabilidad Social como término más amplio y general, emplearemos RS.

ii En Castillo, A. (2009, p.15).

iii En Seitel (2002, p.223)

iv Definido como "cuestiones potencialmente emergentes que pueden aparecer en cualquier momento e influir negativamente en el desarrollo de una organización" (Losada, 2010, p. 54).

${ }^{\vee}$ Entendiendo por comunicación de crisis "la capacidad de una organización de reducir o prever los factores de riesgo e incertidumbre respecto al futuro, de forma que se capacite a la misma para asumir de manera rápida y eficaz las operaciones de comunicación necesarias que contribuyan a reducir o eliminar los efectos negativos que una crisis puede provocar sobre su imagen y reputación" (González Herrero, 2008, p.38).

vi En Lucas (1997, p.103).

vii En Seitel (2002, p.12).

viii Se toma como referente la Capitalización Bursátil como referencia del volumen de negocio de las empresas estudiadas.

${ }^{\text {ix }}$ Debemos aclarar que la empresa Arcelor Mittal, es una empresa internacional que cotiza en la bolsa española por lo que analizaremos su web corporativa como empresa internacional, ya que la web corporativa española es una web comercial y nos ofrece ninguna información relevante para nuestro estudio.

${ }^{x}$ En González Herrero, A. (2006, p.10)

\section{Forma de citar este artículo en bibliografías}

RUIZ-MORA, I. y OLMEDO-SALAR, S. (2013) "Responsabilidad social y prevención de riesgos potenciales. Estudio de las grandes empresas españolas " se encuentra publicado en Revista PANGEA (ISSN: 2172 - 3168); Año 2013, № 2, en páginas 127 a 146. Red Académica Iberoamericana de Comunicación. Recuperado el _ de de de 2 de: http://www.revistapangea.org 